\title{
Microbial Profiles and Risk Factors of Preexisting Biliary Infection in Patients with Therapeutic Endoscopy
}

\author{
Hua-Qiang Ruan, ${ }^{1}$ Guo-Lin Liao, ${ }^{1}$ Peng Peng, ${ }^{1}$ Shi-Quan Liu $\left(D,{ }^{1}\right.$ Chang-Liang Wu, ${ }^{1}$ \\ Jian-Fu Qin, ${ }^{1}$ Zhi-Hai Liang, ${ }^{2}$ Guo-Du Tang, ${ }^{2}$ Meng-Bin Qin $\mathbb{C}^{,},{ }^{1}$ and Jie-An Huang ${ }^{1}$ \\ ${ }^{1}$ Department of Gastroenterology, The Second Affiliated Hospital of Guangxi Medical University, Nanning 530007, China \\ ${ }^{2}$ Department of Gastroenterology, The First Affiliated Hospital of Guangxi Medical University, Nanning 530021, China \\ Correspondence should be addressed to Meng-Bin Qin; dr.mmbin@hotmail.com and Jie-An Huang; hjagxmu@163.com
}

Received 18 December 2018; Accepted 26 March 2019; Published 5 May 2019

Academic Editor: Roberto Caronna

Copyright (c) 2019 Hua-Qiang Ruan et al. This is an open access article distributed under the Creative Commons Attribution License, which permits unrestricted use, distribution, and reproduction in any medium, provided the original work is properly cited.

\begin{abstract}
Background. The bile infection may already exist before the administration of an interventional procedure, despite no clinical manifestations of cholangitis detected. Blood cultures remained negative even in more than half of the febrile cases with cholangitis. Risk factors associated with bacterial growth in bile before the intervention are not well defined. To establish the bacterial profiles isolated from the bile samples and to identify risk factors for bacterial colonization in the bile system. Methods. Individuals who underwent endoscopic retrograde cholangiopancreatography (ERCP) interventions were enrolled. Bile samples were aspirated and were immediately transferred into a sterile tube for storage. Results. Positive bile cultures were detected in 363 (38.0\%) of 956 patients, including 322 benign diseases and 41 malignances. Of 363 positive cases, 351 (96.7\%) were monoinfection and $12(3.3 \%)$ multi-infection. Escherichia coli were the most common Gram-negative bacteria (210, $56.0 \%)$, followed by Klebsiella pneumoniae (45, 12.0\%). Enterococcus faecalis represented the most common Gram-positive microorganism $(19,5.07 \%)$, while Candida albicans $(11,2.93 \%)$ were the dominant fungi. Klebsiella pneumoniae were more frequently detected in malignant diseases $(P=0.046)$. Age, previous ERCP history or OLT history, and CBD diameter were independent risk factors for positive cultures $(P<0.05)$ while preoperative jaundice drug therapy was the protective factor for bile infection $(P<0.05)$. Conclusion. Monomicrobial infection was dominant among all infections, and Klebsiella pneumoniae strains were more frequently isolated from patients with malignant diseases. To effectively manage patients who are at a high risk for bile infection, a detailed diagnosis and treatment plan for each case should be prepared.
\end{abstract}

\section{Introduction}

The bile duct is typically maintained sterile by the continuous flushing action of bile and the bacteriostatic effects of bile salts [1]. Under the conditions of normal bile flow, positive bile culture is not expected. However, bacteria could remain, colonize, and replicate in a relatively stagnant bile environment if a biliary obstruction exists resulting in the increase of pressure. Eventually, the bacteria would spread into the blood and cause systemic infections posing grave consequences.

Endoscopic retrograde cholangiopancreatography (ERCP) has gradually become an indispensable procedure in the diagnosis and treatment of many pancreaticobiliary disorders since its first introduction in the 1970s. Despite a safe and effective record for ERCP, endoscopists must have a thorough understanding of potential adverse events associated with the procedure. Cholangitis is a common adverse event linked to the ERCP procedure [2]. About $0.5 \%$ to $3 \%$ of ERCP cases [3-8] or $0.35 \%$ to $2.4 \%$ of ERCP cases in China $[9,10]$ developed cholangitis after the procedure.

Previous studies suggested incomplete drainage in an obstructed biliary system resulting from choledocholithiasis and incomplete stone clearance were the main risk factors for post-ERCP cholangitis (PEC) [11], especially after the contrast injection. Biliary pathological changes are often secondary to a bacterial colonization postprocedure [12] The infection of the biliary tract causing bacteremia may 


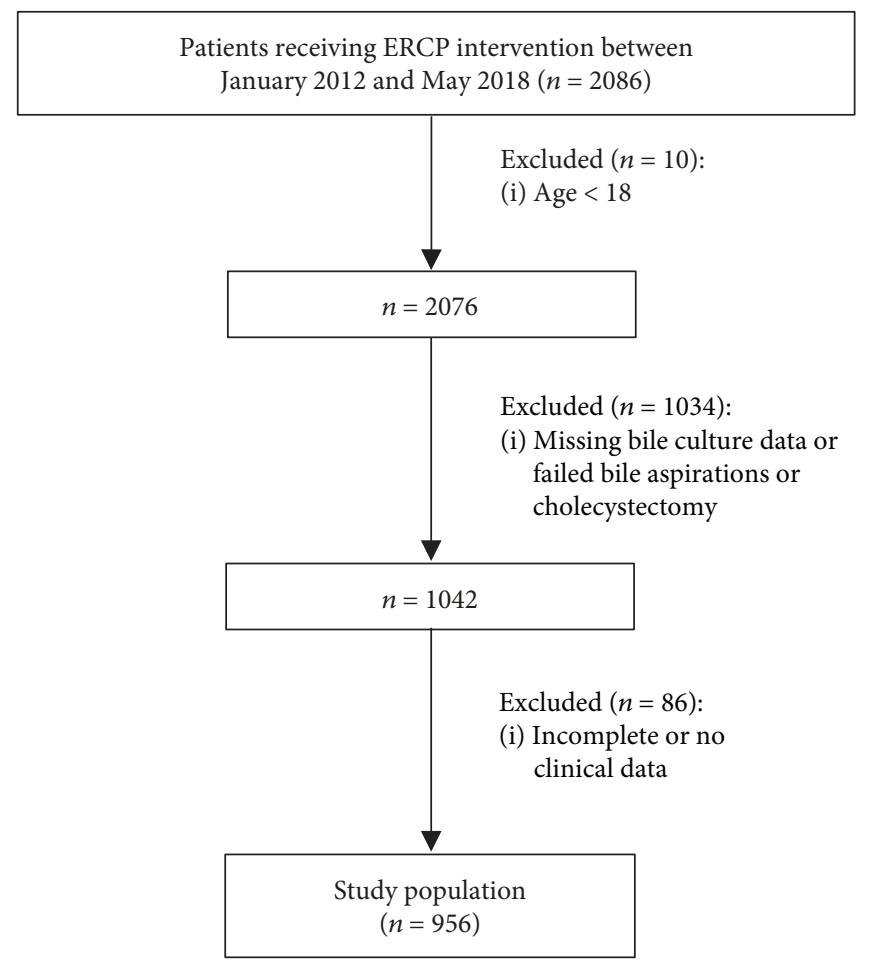

Figure 1: Patient selection flow chart.

manifest as a compatible clinical syndrome, and a blood culture isolate profile may reflect the original infection of cholangitis. However, the infection potentially exists before the ERCP procedure despite lacking the typical manifestations of cholangitis (jaundice, fever, and right upper quadrant pain) $[13,14]$. Blood cultures remained negative in more than half of the febrile cases with cholangitis [15]. Currently, the risk factors for bacterial growth in bile before the intervention have not been clearly defined [16, 17].

The aim of this study is to establish the bacterial profiles isolated from the bile samples and their contributions to the underlying diseases. Furthermore, we investigate the risk factors for microbiological colonization in patients with different biliary diseases.

\section{Methods}

2.1. Patients. Patients who had various biliary or pancreatic disorders and received ERCP procedures between January 2012 and May 2018 at the First and Second Affiliated Hospitals of Guangxi Medical University (Nanning, China) were selected for this study. Patients were excluded if bile aspirations failed or bile culture data did not exist, cholecystectomy, under 18 years of age or in the event of incomplete clinical data. The study was approved by the Institutional Review Boards of Hospitals. A written informed consent was obtained from all participants.

2.2. Procedures. All ERCP procedures were administered by well-trained and experienced endoscopists, who could perform the procedures that have the ERCP difficulty at Grade 3 per the ERCP core curriculum [18].

The ERCP interventions were conducted using a therapeutic duodenoscope (TJF-260V; Olympus Optical, Tokyo, Japan). The selective cannulation was performed via the common bile duct (CBD) by using a guidewire or a standard catheter for cases with a preexisting sphincterotomy. All duodenoscopes were disinfected according to the guidelines and decontaminated, assessed by regular smear tests. Once the duodenoscope entered successfully and guidewire cannulation was established, bile was aspirated by inserting a single use, 5F, standard sphincterotome catheter into the bile duct before the injection of a contrast agent for the ERCP procedure. Approximately 2 to $8 \mathrm{~mL}$ of bile (average $4 \mathrm{~mL}$ ) was collected and immediately transferred into a sterile tube.

2.3. Observational Index. The demographics and clinical information before the cholangiopancreatography were collected including patients' gender and age, endoscopic diagnoses, previous ERCP history, preoperative jaundice drug therapy, Billroth II gastrectomy history, orthotopic liver transplantation (OLT) history, common bile duct diameter, and papilla types. Patterns of cannulation of univariate and multivariate analysis were performed to identify the independent risk factors of bacterial colonization in bile.

2.4. Statistical Analysis. Continuous variables were expressed as the mean and standard deviation (s.d.) or the median and IQR. Counts and percentages were determined if appropriate. The categorical variables were analyzed by Pearson's chi-square test or Fisher's exact test. In univariate analysis, the test level was unrestricted to 0.10 if the covariates 
TABle 1: Baseline demographics and endoscopic features of enrolled patients.

\begin{tabular}{lc}
\hline Study population & 956 \\
\hline Mean age (years, s.d.) & $58.4 \pm 15.0$ \\
Females (\%) & $38.70 \%$ \\
Benign diseases & $835(87.3 \%)$ \\
Malignant diseases & $121(12.7 \%)$ \\
Endoscopic diagnoses & \\
$\quad$ Normal cholangiopancreatography & $21(2.2 \%)$ \\
Cholelithiasis & $694(72.6 \%)$ \\
Malignant strictures & $121(12.7 \%)$ \\
Benign strictures & $58(6.1 \%)$ \\
Bile duct expansions for unknown reasons & $39(4.1 \%)$ \\
Pancreatic disorders & $5(0.5 \%)$ \\
Clonorchiasis & $9(0.9 \%)$ \\
Other diseases & $9(0.9 \%)$ \\
\hline
\end{tabular}

exhibited a high level of significance. Multivariate regression analyses were used to identify various risk factors. Logistic regression models were employed to calculate odds ratios with 95\% confidence intervals (CIs). A two-tailed $P$ value < 0.05 was considered statistically significant (SPSS 22.0 for Windows, SPSS, Chicago, IL).

\section{Results}

3.1. General Characteristics. This retrospective study initially screened 2086 consecutive patients who underwent ERCP in the two hospitals between January 2012 and May 2018. The information contained in medical charts, computerized records, and image studies was retrieved. Patients were excluded if under 18 years of age $(n=10)$, no bile culture data, failed bile aspirations or cholecystectomy $(n=1034)$, or incomplete clinical data $(n=86)$. Note that the bile collection is not generally recommended by gastroenterology societies due to being technically demanding, time consuming, or redundant in the presence of blood cultures. Thus, the bile collection is an option under the ERCP procedure and contingent upon various indications, resulting in no bile collections in a considerable number of patients with ERCP procedures [17]. In addition, patients were also excluded because of inadequate bile aspiration. A total of 956 patients were finally enrolled and analyzed (Figure 1). The mean age was $58.4 \pm 15.0$ years (range: $18-92$ years), and $61.3 \%$ of the cohort were male. Among the 956 patients, 835 had benign diseases and 121 with malignances. All patients with acute cholangitis were treated with antibiotics prior to cholangiography.

The endoscopic diagnosis consisted of 694 cholelithiasis (72.6\%), 121 malignant strictures (12.7\%), 58 benign strictures $(6.1 \%), 39$ bile duct expansions for unknown reasons (4.1\%), five pancreatic disorders $(0.5 \%), 21$ normal cholangiopancreatography $(2.2 \%)$, nine clonorchiasis $(0.9 \%)$, and nine other diseases $(0.9 \%)$, which included six congenital choledochal cyst, two biliary fistulas after surgeries, and one sphincter of Oddi dysfunction (SOD) (Table 1).
TABle 2: Microbiological classification of positive bile culture.

\begin{tabular}{|c|c|c|}
\hline Organisms & $\mathrm{N}$ & $\%$ \\
\hline \multicolumn{3}{|l|}{ Bacteria } \\
\hline Gram-positive & 51 & 13.6 \\
\hline Enterococcus spp. & 43 & 11.5 \\
\hline Enterococcus faecalis & 19 & 5.1 \\
\hline Enterococcus faecium & 14 & 3.7 \\
\hline Enterococcus casselifavus & 7 & 1.9 \\
\hline Other Enterococcus species & 3 & 0.8 \\
\hline Streptococcus spp. & 6 & 1.6 \\
\hline Streptococcus hemolyticus & 4 & 1.1 \\
\hline Other Streptococcus species & 2 & 0.5 \\
\hline Staphylococcus spp. & 2 & 0.5 \\
\hline Gram-negative & 293 & 78.1 \\
\hline Escherichia coli & 210 & 56.0 \\
\hline Klebsiella spp. (3 K. oxytoca included) & 48 & 12.8 \\
\hline Pseudomonas spp. & 9 & 2.4 \\
\hline Enterobacter cloacae & 9 & 2.4 \\
\hline Citrobacterium spp. & 4 & 1.1 \\
\hline Serratia fonticola & 4 & 1.1 \\
\hline Aeromonas hydrophila & 4 & 1.1 \\
\hline Flavobacterium spp. & 2 & 0.5 \\
\hline Proteobacteria spp. & 2 & 0.5 \\
\hline Morganella Fulton & 1 & 0.3 \\
\hline \multicolumn{3}{|l|}{$M D R s$} \\
\hline Acinetobacter baumannii & 4 & 1.1 \\
\hline Acinetobacter lwoffii & 1 & 0.3 \\
\hline \multicolumn{3}{|l|}{ Fungi } \\
\hline Candida albicans & 11 & 2.9 \\
\hline Candida tropicalis & 7 & 1.9 \\
\hline Candida glabrata & 5 & 1.3 \\
\hline Candida cornea & 3 & 0.8 \\
\hline
\end{tabular}

3.2. Microbiological Characteristics in Bile Culture. Positive bile cultures were detected in 363 (38.0\%) of 956 patients, including 322 with benign diseases and 41 with malignant diseases. Of 363 positive samples, 351 (96.7\%) were single bacterial infection and $12(3.3 \%)$ were mixed infection. Further, a total of 34 species and 375 strains of microorganisms were identified (Table 2). There were 298 strains of Gram-negative bacteria, 51 strains of Gram-positive bacteria, and 26 strains of fungi. Five strains of Multidrug-Resistant Organisms (MDRs) were identified including four Acinetobacter baumannii and one Acinetobacter lwoffi. Among the 298 Gram-negative strains, the most common ones were Escherichia coli (210, 56.0\%), Klebsiella pneumoniae (45, 12.0\%), Pseudomonas aeruginosa (8, 2.13\%), and Enterobacter cloacae $(8,2.13 \%)$. The most common Grampositive bacteria were Enterococcus faecalis (19, 5.07\%) and Enterococcus faecium (14, 3.73\%). The most common fungi were Candida albicans (11, 2.93\%) and Candida tropicalis $(7,1.87 \%)$. 
TABLE 3: Bacterial composition between benign and malignant diseases.

\begin{tabular}{lcccc}
\hline Species & Benign $(n=322)(\%)$ & Malignant $(n=41)(\%)$ & $P$ & Total $(n=363)(\%)$ \\
\hline Escherichia coli & $189(58.7)$ & $21(51.2)$ & 0.456 & $210(57.9)$ \\
Klebsiella spp. & $38(11.8)$ & $10(24.4)$ & $\mathbf{0 . 0 4 6}$ & $48(13.2)$ \\
Enterococcus spp. & $35(10.9)$ & $8(19.5)$ & 0.175 & $43(11.8)$ \\
Fungi & $16(5.0)$ & $3(7.3)$ & 0.792 & $19(5.2)$ \\
\hline
\end{tabular}

In addition, we investigated the four most prevalent microorganism distributions in benign and malignant diseases. A greater number of Klebsiella pneumoniae strains were detected in malignant diseases compared to those found in benign diseases $(22.4 \%$ vs. $11.8 \%, P=0.046)$, while there were no significant differences in the numbers of strains of Escherichia coli $(58.7 \%$ vs. $51.2 \%, P=0.456)$, Enterococcus species $(10.9 \%$ vs. $19.5 \%, P=0.175)$, and fungi $(5.0 \%$ vs. $7.3 \%, P=0.792)$ between benign and malignant disease groups (Table 3 ).

3.3. Risk Factors for Bacterial Colonization in Bile. Univariate analysis demonstrated that age, previous history of ERCP, previous orthotopic liver transplantation, preoperative jaundice drug therapy, various endoscopic diagnoses, common bile duct diameter, different papilla types, and patterns of cannulation were all risk factors for positive bile culture $(P<0.10)$ (Table 4$)$.

The variables identified through the univariate analysis and additional factors selected based on clinical experience and the literature were included in a multivariate logistic regression analysis. Our multivariate analysis established age, previous history of ERCP, previous history of orthotopic liver transplantation, and common bile duct diameter as independent risk factors for positive bile culture $(P<0.05)$ (Table 5). Nevertheless, preoperative jaundice drug therapy is classified as a protective factor against positive bile culture $(P<0.05)$.

\section{Discussion}

A normal sterile and free flow biliary system is not a favorable environment for bacterial growth. However, bile secretion can be restricted or blocked within the bile duct due to biliary obstruction. The bacteria that are transferred into the bile duct through the duodenal papilla can reside in the bile duct under the restricted bile flow scenario, ultimately replicating, colonizing, and causing biliary infection with severe consequences [19].

In this study, we identified the microbial profiles in infected bile systems. The positive bile culture rate was $38.0 \%$ among the 956 patients analyzed. Gram-positive and Gram-negative bacteria accounted for $13.6 \%$ and $78.1 \%$, respectively, with the remaining $7 \%$ fungi positive. Escherichia coli was the most common Gram-negative bacteria, followed by Klebsiella pneumoniae, Pseudomonas aeruginosa, and Enterobacter cloacae. Enterococcus species represented the most common Gram-positive bacteria. The composition of biliary pathogens was consistent with other studies, resembling the intestinal bacterial flora $[14,20]$. In a large study cohort involving 509 consecutive individuals who underwent early laparoscopic cholecystectomy (within 72 hours) or percutaneous cholecystostomy, 171 (33.6\%) of them tested bile culture positive. Gram-negative organisms accounted for $80.1 \%$ (137/171). Among them, E. coli was the most frequent isolate while Enterococcus was the most common Gram-positive sample [21]. Other independent studies reported $16 \%$ to $85 \%$ positive bile culture among different disease groups [14, 15, 17, 22-25].

The detected monomicrobial infection was more frequently $(351 / 363,96.7 \%)$ compared with multimicrobial infection $(12 / 363,3.3 \%)$. Kaya et al. [14] reported that a single bacterobilia accounted for $95 \%$ of infection. However, higher multi-infection rates compared to monoinfection rates were reported in other studies $[17,26]$. Overprescription of antibiotics before hospitalization in China or suboptimal culture conditions may explain the differences. Monomicrobial infection, especially with Gram-negative bacterium, was frequently associated with patients requiring repeated ERCP interventions. This finding provides guidance to select an initial antibiotic regimen.

Published studies suggested a trend of higher infection rates of Helicobacter spp. in individuals with malignant bile duct diseases compared to normal controls or those with benign biliary diseases [27]. Thus, we analyzed the distribution of the identified organisms between our benign and malignant groups. In the benign pancreaticobiliary diseased group, Escherichia coli strains were dominant, followed by Klebsiella pneumoniae and Enterococcus spp. A similar trend was noted in the malignant group. These results may guide physicians to select more efficacious antimicrobials. However, the infection with Klebsiella spp. was significantly higher in malignant biliary diseases $(P=0.046$, Table 3$)$. Other studies [28, 29] also reported a higher culture rate of the Klebsiella pneumoniae strain among malignant diseases. Klebsiella spp. (mainly Klebsiella pneumoniae) is frequently found in human intestines and the upper respiratory tract causing opportunistic infections. The antibacterial effectiveness can be hampered by antibiotic resistance. It was noteworthy that malignant diseases appeared more frequently in individuals aged 60 years or older compared to those younger patients.

Unrestricted cancer growth deprives the body of proper nutrition, injures the mucosal barrier, compromises immunity function, and ultimately leads to infection. The microorganism profiles isolated from the bile samples of malignant diseases differed from those described in the Tokyo Guidelines and other studies of benign biliary 
TABLE 4: Univariate analysis of risk factors for bile infection.

\begin{tabular}{|c|c|c|c|c|}
\hline Factors & Positive culture $(n)$ & Negative culture $(n)$ & $\chi^{2}$ & $P$ value \\
\hline \multicolumn{5}{|l|}{ Gender } \\
\hline Male & 228 & 358 & 0.466 & 0.495 \\
\hline Female & 135 & 235 & & \\
\hline \multicolumn{5}{|l|}{ Age } \\
\hline$\geq 60$ years & 226 & 256 & 32.061 & $<0.001$ \\
\hline$<60$ years & 137 & 337 & & \\
\hline \multicolumn{5}{|l|}{ Previous ERCP history } \\
\hline Yes & 145 & 129 & 35.558 & $<0.001$ \\
\hline No & 218 & 464 & & \\
\hline \multicolumn{5}{|l|}{ Previous Billroth II history } \\
\hline Yes & 3 & 3 & 0.035 & 0.852 \\
\hline No & 360 & 590 & & \\
\hline \multicolumn{5}{|l|}{ Previous OLT } \\
\hline Yes & 13 & 5 & 7.716 & 0.005 \\
\hline No & 359 & 588 & & \\
\hline \multicolumn{5}{|l|}{ Preoperative jaundice drug therapy } \\
\hline Yes & 161 & 315 & 6.577 & 0.010 \\
\hline No & 202 & 278 & & \\
\hline \multicolumn{5}{|l|}{ Endoscopic diagnoses } \\
\hline Normal cholangiopancreatography & 1 & 20 & 17.719 & 0.012 \\
\hline Cholelithiasis & 276 & 418 & & \\
\hline Malignant strictures & 41 & 80 & & \\
\hline Benign strictures & 27 & 31 & & \\
\hline Bile duct expansions for UR & 12 & 27 & & \\
\hline Pancreatic disorders & 1 & 4 & & \\
\hline Other diseases & 4 & 5 & & \\
\hline Clonorchiasis & 1 & 8 & & \\
\hline \multicolumn{5}{|l|}{ Common bile duct diameters } \\
\hline$\geq 12 \mathrm{~mm}$ & 284 & 344 & 39.981 & $<0.001$ \\
\hline$<12 \mathrm{~mm}$ & 79 & 249 & & \\
\hline \multicolumn{5}{|l|}{ Papilla types } \\
\hline Normal & 221 & 460 & 41.289 & $<0.001$ \\
\hline Minor papilla & 5 & 17 & & \\
\hline Papillary diverticulum & 104 & 77 & & \\
\hline Papillary carcinoma & 20 & 23 & & \\
\hline Papillary fistula & 13 & 16 & & \\
\hline \multicolumn{5}{|l|}{ Patterns of cannulation } \\
\hline Routine & 353 & 557 & 5.412 & 0.067 \\
\hline Dual-guidewire & 6 & 21 & & \\
\hline Precut papillotomy & 4 & 15 & & \\
\hline
\end{tabular}

OLT: orthotopic liver transplantation. Bile duct expansions for UR: unknown reasons. Papillary precut: a needle-like knife was used to cut layer by layer from 11 o'clock position of the papillary uplift highest point to papillary openings, or a needle-like knife was vertically used to pierce and fenestrate via the highest point of the papillary highest bump.

disorders. This knowledge provides an alternative solution when selecting empirical antibiotics prior to the availability of a positive culture or after a negative culture.

The multivariate study identified old age as an independent risk factor for positive bile cultures. Mahafzah and Daradkeh reported that the percentage of positive cultures increased with age [30]. It is highly recommended that the elderly patients should be adequately evaluated for bile infection before the intervention.

This study found that previous ERCP history or OLT history was associated with positive cultures. A significantly higher bacteriobilia was observed in patients who required 
TABLE 5: Multivariate analysis of risk factors for positive bile culture.

\begin{tabular}{|c|c|c|c|c|}
\hline Factors & Wald & OR & $P$ value & $95 \% \mathrm{CI}$ \\
\hline Preoperative jaundice drug therapy & 5.267 & 0.704 & 0.022 & $0.521-0.950$ \\
\hline Age & 9.267 & 1.592 & 0.002 & $1.180-2.147$ \\
\hline Common bile duct diameter & 19.629 & 2.114 & $<0.001$ & $1.518-2.944$ \\
\hline Endoscopic diagnoses & 5.819 & & 0.561 & \\
\hline Cholelithiasis & 0.246 & 0.475 & 0.620 & $0.025-8.957$ \\
\hline Malignant strictures & 0.654 & 2.414 & 0.419 & $0.285-20.450$ \\
\hline Benign strictures & 0.457 & 2.124 & 0.499 & $0.239-18.845$ \\
\hline Bile duct expansions for unknown reasons & 0.659 & 2.505 & 0.417 & $0.273-23.017$ \\
\hline Pancreatic disorders & 0.320 & 1.915 & 0.572 & $0.201-18.211$ \\
\hline Other diseases & 0.000 & 1.026 & 0.987 & $0.046-22.797$ \\
\hline Clonorchiasis & 1.905 & 5.889 & 0.168 & $0.475-73.035$ \\
\hline Previous ERCP history & 22.250 & 2.156 & $<0.001$ & $1.567-2.967$ \\
\hline Previous orthotopic liver transplantation & 7.198 & 4.914 & 0.007 & $1.536-15.725$ \\
\hline Papilla types & 24.076 & & 0.000 & \\
\hline Minor papilla & 0.172 & 0.846 & 0.678 & $0.383-1.868$ \\
\hline Papillary diverticulum & 0.587 & 0.599 & 0.444 & $0.162-2.220$ \\
\hline Tumor & 2.960 & 2.082 & 0.085 & $0.903-4.799$ \\
\hline Papillary fistula & 0.171 & 1.254 & 0.679 & $0.429-3.662$ \\
\hline Cannulation methods & 3.486 & & 0.175 & \\
\hline Dual-guidewire & 1.685 & 2.167 & 0.194 & $0.674-6.962$ \\
\hline Precut papillotomy & 0.013 & 1.093 & 0.908 & $0.244-4.890$ \\
\hline
\end{tabular}

repeated interventions and orthotopic liver transplantation, as reported in a study cohort with 243 consecutive patients who underwent ERCP or percutaneous transhepatic cholangiography (PTC) [17]. Yun and Seo [31] suggested the bile of patients with laparoscopic cholecystectomy may contain microorganisms, particularly in those who received repeated ERCP. Interventions, like Oddi's sphincterotomy, breakdown the normal human defense mechanisms, decrease the pressure of the bile duct, and potentially cause a reverse fluid flow from the duodenum into the bile duct $[9,26]$. The individuals who underwent these interventions might have acquired biliary tract motor dysfunction and are prone to biliary infection. Furthermore, the bile duct at anastomosis location after surgery like OLT could become narrowed, and the anatomical structures could be altered. With the reflux of bile increasing, biliary mucosa could experience inflammatory edema, ultimately generating a favorable environment for bacterial proliferation and colonization.

Common bile duct diameters at $\geq 12 \mathrm{~mm}$ were also determined as an independent risk factor, which has been suggested to be an important risk factor for post-ERCP cholangitis with biliary type sphincter of Oddi dysfunction [32], especially in type I. However, it remains unclear how CBD diameter triggers the positive bile culture before the ERCP interventions. Long-term disease courses, repeated inflammatory stimulations, large $\mathrm{CBD}$ stones and distal stricture caused by benign or malignant diseases may induce the bile duct obstruction or dilatation. Consequently, an increased biliary pressure traps and retains microorganisms within the stagnant bile.
We noted a higher tendency of positive bile culture in patients with papillary carcinoma in this analysis. Papillary carcinoma may cause malignant biliary obstruction as well as biliary infection, and a combination of both might have a profound effect on clinical outcomes, as well as the quality of life [33].

Interestingly, preoperative jaundice drug therapy was identified as an independent protective factor for the absence of infection. The alleviated jaundice therapy may improve biliary drainage and decrease the retention of organisms in the bile duct.

The use of prophylactic antibiotics before the ERCP procedure is not routinely required, according to the guidelines updated by the Digestive Endoscopy Branch of British Society of Digestion (BSG) in June 2009. A full antibiotic course is required when the treatment is aimed at achieving adequate biliary decompression by repeat interventions [34]. Currently, the third-generation cephalosporins or penicillin/ $\beta$-lactamase inhibitors are recommended as the empirical treatment for biliary infections [35]. The bile culture is invaluable in the selection of efficacious and appropriate antibiotics for the treatment of biliary infections.

To avoid cross-transmission among different patients, the facilities and instruments were maintained at a sterilized status. We have followed stringent standards to conduct all ERCP procedures. We set the detection of microorganisms at a level of 10,000 per $\mathrm{mL}$ of bile as positive, and anything below this level was deemed negative $[14,17]$. We are confident in the validity of the bile culture results. However, we could not completely exclude the possibility of bile sample 
contamination during which the duodenoscope was passed through the gastrointestinal tract.

Additional limitations include the following: an inherent selection bias associated with a retrospective study; the lack of confirmation of positive bile cultures by blood culture; and the need for multicenter different region-based studies.

In conclusion, our results show that $38 \%$ of patients with various biliary or pancreatic disorders had bacteriobilia. The most commonly isolated bacteria were Gram-negative bacteria including Escherichia coli and Klebsiella pneumoniae. Monomicrobial infection was more prevalent compared with multimicrobial infection. A higher percentage of Klebsiella pneumoniae strains was detected in the malignant diseases compared to the benign group. The identified risk factors associated with positive bile culture included old age, previous history of ERCP or OLT, and larger common bile duct diameter. A preoperative jaundice drug therapy was a factor associated with negative bile culture. An implication of our findings recommends that preoperative precaution should be adopted and a detailed management plan should be prepared in advance, considering about one-third of patients likely have had bile infection, previously. Bile samples should be collected for culture to confirm potential infection whenever possible.

\section{Data Availability}

The data used to support the findings of this study are available from the corresponding authors upon request.

\section{Ethical Approval}

This study was approved by the Ethics Committee of the Second Affiliated Hospital of Guangxi Medical University, Nanning, China.

\section{Conflicts of Interest}

No benefits in any form have been received or will be received from a commercial party related directly or indirectly to the subject of this article.

\section{Authors' Contributions}

Qin MB and Huang JA proposed the study. Ruan HQ performed the research and wrote the manuscript. Ruan HQ, Liao GL, Peng P, Liu SQ, Wu CL, Qin JF, Liang ZH, Tang $\mathrm{GD}$, and Qin MB collected and analyzed the data. All authors contributed to the design and interpretation of the results and to the revision. Ruan HQ, Liao GL, Qin MB, and Huang JA contributed equally to this study. Qin MB and Huang JA were the guarantors and corresponding authors.

\section{References}

[1] J. Y. Sung, J. W. Costerton, and E. A. Shaffer, "Defense system in the biliary tract against bacterial infection," Digestive Diseases and Sciences, vol. 37, no. 5, pp. 689696, 1992.
[2] ASGE Standards of Practice Committee, V. Chandrasekhara, M. A. Khashab et al., "Adverse events associated with ERCP," Gastrointestinal Endoscopy, vol. 85, no. 1, pp. 3247, 2017.

[3] E. Masci, G. Toti, A. Mariani et al., "Complications of diagnostic and therapeutic ERCP: a prospective multicenter study," The American Journal of Gastroenterology, vol. 96, no. 2, pp. 417-423, 2001.

[4] A. Andriulli, S. Loperfido, G. Napolitano et al., "Incidence rates of post-ERCP complications: a systematic survey of prospective studies," The American Journal of Gastroenterology, vol. 102, no. 8, pp. 1781-1788, 2007.

[5] O. Barkay, M. Khashab, M. Al-Haddad, and E. L. Fogel, "Minimizing complications in pancreaticobiliary endoscopy," Current Gastroenterology Reports, vol. 11, no. 2, pp. 134141, 2009.

[6] J. B. Colton and C. C. Curran, "Quality indicators, including complications, of ERCP in a community setting: a prospective study," Gastrointestinal Endoscopy, vol. 70, no. 3, pp. 457467, 2009.

[7] S. Ismail, L. Kylänpää, H. Mustonen et al., "Risk factors for complications of ERCP in primary sclerosing cholangitis," Endoscopy, vol. 44, no. 12, pp. 1133-1138, 2012.

[8] C. Kapral, A. Mühlberger, F. Wewalka et al., "Quality assessment of endoscopic retrograde cholangiopancreatography: results of a running nationwide Austrian benchmarking project after 5 years of implementation," European Journal of Gastroenterology \& Hepatology, vol. 24, no. 12, pp. 1447$1454,2012$.

[9] M. Chen, L. Wang, Y. Wang et al., "Risk factor analysis of postERCP cholangitis: a single-center experience," Hepatobiliary \& Pancreatic Diseases International, vol. 17, no. 1, pp. 5558, 2018.

[10] Z. S. Li, G. M. Xu, Z. X. Sun, X. P. Zhou, Z. D. Jin, and D. W. Zhou, "Early complications of diagnostic and therapeutic ERCP and its treatment," Chinese Journal of Digestive Endoscopy, vol. 19, no. 2, pp. 77-80, 2002.

[11] M. L. Freeman, "Complications of endoscopic biliary sphincterotomy: a review," Endoscopy, vol. 29, no. 4, pp. 288 297, 1997.

[12] A. Csendes, M. Fernandez, and P. Uribe, "Bacteriology of the gallbladder bile in normal subjects," The American Journal of Surgery, vol. 129, no. 6, pp. 629-631, 1975.

[13] M. Melzer, R. Toner, S. Lacey, E. Bettany, and G. Rait, "Biliary tract infection and bacteraemia: presentation, structural abnormalities, causative organisms and clinical outcomes," Postgraduate Medical Journal, vol. 83, no. 986, pp. 773776, 2007.

[14] M. Kaya, R. Beştaş, F. Bacalan, F. Bacaksız, E. G. Arslan, and M. A. Kaplan, "Microbial profile and antibiotic sensitivity pattern in bile cultures from endoscopic retrograde cholangiography patients," World Journal of Gastroenterology, vol. 18, no. 27, pp. 3585-3589, 2012.

[15] R. Rerknimitr, E. L. Fogel, C. Kalayci, E. Esber, G. A. Lehman, and S. Sherman, "Microbiology of bile in patients with cholangitis or cholestasis with and without plastic biliary endoprosthesis," Gastrointestinal Endoscopy, vol. 56, no. 6, pp. 885-889, 2002.

[16] C. Arminanzas, L. A. Herrera, and M. C. Farinas, "Bacteriobilia: a non-resolved problem," Revista Española de Quimioterapia, vol. 29, no. 3, pp. 113-118, 2016. 
[17] A. A. Negm, A. Schott, R. P. Vonberg et al., "Routine bile collection for microbiological analysis during cholangiography and its impact on the management of cholangitis," Gastrointestinal Endoscopy, vol. 72, no. 2, pp. 284-291, 2010.

[18] ASGE Training Committee, J. Jorgensen, N. Kubiliun et al., "Endoscopic retrograde cholangiopancreatography (ERCP): core curriculum," Gastrointestinal Endoscopy, vol. 83, no. 2, pp. 279-289, 2016.

[19] E. Ortiz-Brizuela, J. Sifuentes-Osornio, D. Manzur-Sandoval et al., "Acute cholangitis after bilioenteric anastomosis for bile duct injuries," Journal of Gastrointestinal Surgery, vol. 21, no. 10, pp. 1613-1619, 2017.

[20] T. Voigtländer, E. Leuchs, R. P. Vonberg et al., "Microbiological analysis of bile and its impact in critically ill patients with secondary sclerosing cholangitis," Journal of Infection, vol. 70, no. 5, pp. 483-490, 2015.

[21] Y. B. Hadi, M. Waqas, H. M. Umer et al., "Bacterobilia in acute cholecystitis: bile cultures' isolates, antibiotic sensitivities and antibiotic usage. A study on a Pakistani population," JPMA: Journal of Pakistan Medical Association, vol. 66, no. 10, Supplement 3, pp. S50-S52, 2016.

[22] J. Sakata, Y. Shirai, Y. Tsuchiya, T. Wakai, T. Nomura, and K. Hatakeyama, "Preoperative cholangitis independently increases in-hospital mortality after combined major hepatic and bile duct resection for hilar cholangiocarcinoma," Langenbeck's Archives of Surgery, vol. 394, no. 6, pp. 1065-1072, 2009.

[23] J. Pohl, A. Ring, W. Stremmel, and A. Stiehl, "The role of dominant stenoses in bacterial infections of bile ducts in primary sclerosing cholangitis," European Journal of Gastroenterology \& Hepatology, vol. 18, no. 1, pp. 69-74, 2006.

[24] G. Millonig, T. Buratti, I. W. Graziadei et al., "Bactobilia after liver transplantation: frequency and antibiotic susceptibility," Liver Transplantation, vol. 12, no. 5, pp. 747-753, 2006.

[25] R. Kiesslich, M. Holfelder, D. Will et al., "Interventional ERCP in patients with cholestasis. Degree of biliary bacterial colonization and antibiotic resistance," Zeitschrift für Gastroenterologie, vol. 39, no. 12, pp. 985-992, 2001.

[26] J. R. Alves, R. C. Silva, S. C. P. Guerra, T. T. Freitas, D. L. B. Souza, and E. C. Amico, "Microbiological analysis of bile in patients with benign and malignant biliopancreatic diseases and its consequences," Arquivos de Gastroenterologia, vol. 53, no. 3, pp. 156-162, 2016.

[27] D. Zhou, J. D. Wang, M. Z. Weng et al., "Infections of Helicobacter spp. in the biliary system are associated with biliary tract cancer: a meta-analysis," European Journal of Gastroenterology \& Hepatology, vol. 25, no. 4, pp. 447-454, 2013.

[28] H. Yu, Z. Guo, W. Xing, X. Guo, F. Liu, and B. Li, "Bile culture and susceptibility testing of malignant biliary obstruction via PTBD," CardioVascular and Interventional Radiology, vol. 35, no. 5, pp. 1136-1144, 2012.

[29] S. I. Cuervo, R. Sánchez, J. C. Gómez-Rincón, C. Almenares, J. P. Osorio, and M. J. Vargas, "Comportamiento de casos de Klebsiella pneumoniae productora de carbapenemasas en pacientes con cáncer de un hospital de tercer nivel de Bogotá, D.C.," Biomédica, vol. 34, Supplement 1, pp. 170180, 2014.

[30] A. M. Mahafzah and S. S. Daradkeh, "Profile and predictors of bile infection in patients undergoing laparoscopic cholecystectomy," Saudi Medical Journal, vol. 30, no. 8, pp. 1044-1048, 2009.
[31] S. P. Yun and H. I. Seo, "Clinical aspects of bile culture in patients undergoing laparoscopic cholecystectomy," Medicine, vol. 97, no. 26, article e11234, 2018.

[32] H. Miyatani, H. Mashima, M. Sekine, and S. Matsumoto, "Post-ERCP biliary complications in patients with biliary type sphincter of Oddi dysfunction," Scientific Reports, vol. 8, no. 1, article 9951, 2018.

[33] H. O. Kim, S. I. Hwang, H. Kim, and J. H. Shin, "Quality of survival in patients treated for malignant biliary obstruction caused by unresectable pancreatic head cancer: surgical versus non-surgical palliation," Hepatobiliary \& Pancreatic Diseases International, vol. 7, no. 6, pp. 643-648, 2008.

[34] S. Ishaq and A. Lipp, "Antibiotic prophylaxis in gastrointestinal endoscopy," Gut, vol. 59, no. 9, article 1300, 2010.

[35] Z. Sun, Y. Zhu, B. Zhu, G. Xu, and N. Zhang, "Controversy and progress for treatment of acute cholangitis after Tokyo Guidelines (TG13)," BioScience Trends, vol. 10, no. 1, pp. 22-26, 2016. 


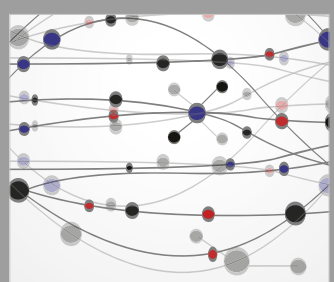

The Scientific World Journal
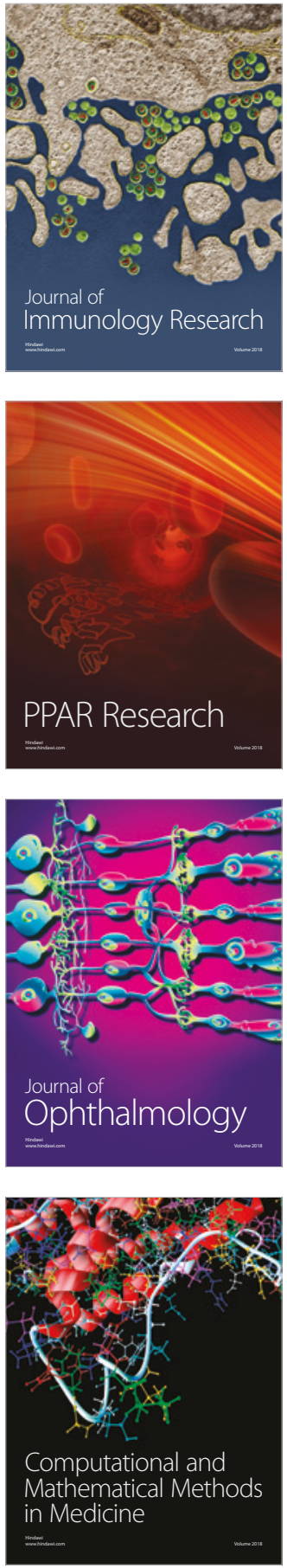

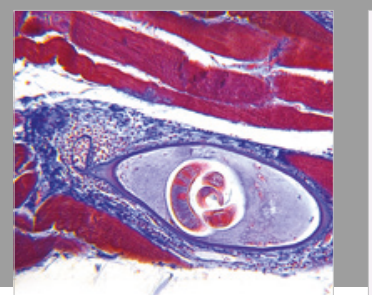

Gastroenterology Research and Practice

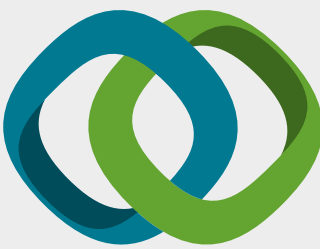

\section{Hindawi}

Submit your manuscripts at

www.hindawi.com
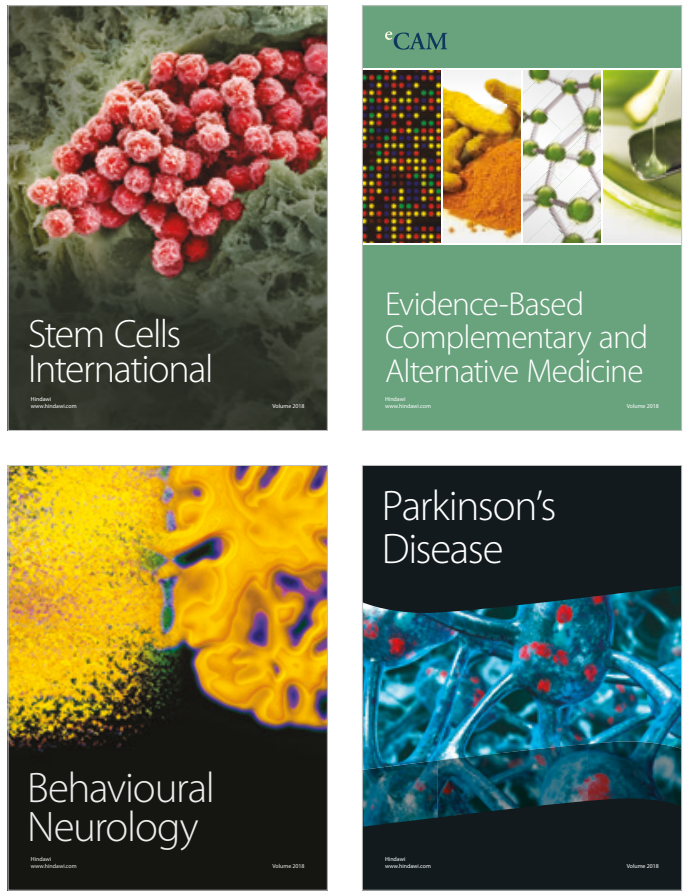

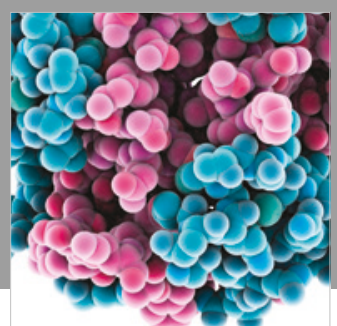

ournal of

Diabetes Research

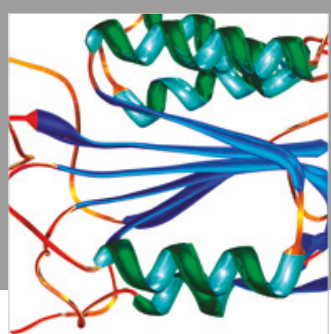

Disease Markers
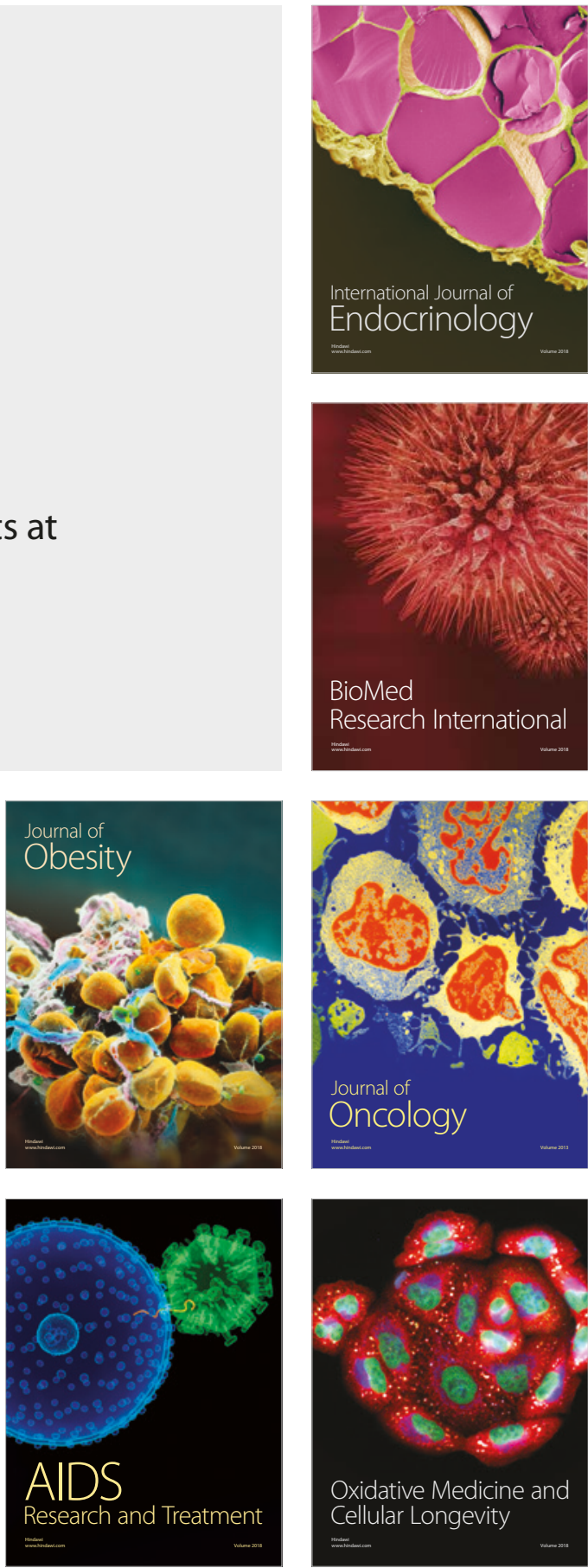